\title{
Geometry of entangled states, Bloch spheres and Hopf fibrations
}

\author{
Rémy Mosseri ${ }^{1}$ and Rossen Dandoloff ${ }^{2}$ \\ ${ }^{1}$ Groupe de Physique des Solides (CNRS UMR 7588), Universités Pierre et Marie Curie Paris 6 \\ et Denis Diderot Paris 7, 2 Place Jussieu, 75251 Paris, Cedex 05, France \\ ${ }^{2}$ Laboratoire de Physique Theorique et Modelisation (CNRS-ESA 8089), Universite de \\ Cergy-Pontoise, Site de Neuville 3, 5 Mail Gay-Lussac, F-95031 Cergy-Pontoise Cedex, \\ France \\ E-mail: mosseri@gps.jussieu.fr and rossen.dandoloff@ptm.u-cergy.fr
}

Received 3 September 2001

Published 16 November 2001

Online at stacks.iop.org/JPhysA/34/10243

\begin{abstract}
We discuss a generalization of the standard Bloch sphere representation for a single qubit to two qubits, in the framework of Hopf fibrations of highdimensional spheres by lower dimensional spheres. The single-qubit Hilbert space is the three-dimensional sphere $S^{3}$. The $S^{2}$ base space of a suitably oriented $S^{3}$ Hopf fibration is nothing but the Bloch sphere, while the circular fibres represent the overall qubit phase degree of freedom. For the two-qubits case, the Hilbert space is a seven-dimensional sphere $S^{7}$, which also allows for a Hopf fibration, with $S^{3}$ fibres and a $S^{4}$ base. The most striking result is that suitably oriented $S^{7}$ Hopf fibrations are entanglement sensitive. The relation with the standard Schmidt decomposition is also discussed.
\end{abstract}

PACS numbers: 03.65., 03.65.Ta, 03.67.- a, 42.50.-p

\section{Introduction}

The interest in two-level systems, or coupled two-level systems, is as old as in quantum mechanics itself, with the analysis of the electron spin sector in the helium atom. The ubiquitous two-level systems have gained a renewed interest in the past ten years, owing to the fascinating perspectives in quantum manipulation of information and quantum computation [1]. These two-level quantum systems are now called qubits, grouped and coupled into q-registers, and manipulated by sophisticated means. It is of interest to describe their quantum evolution in a suitable representation space, in order to get some insight into the subtleties of this complicated problem. A well known tool in quantum optics is the Bloch sphere representation, where the simple qubit state is faithfully represented, up to its 
overall phase, by a point on a standard sphere $S^{2}$, whose coordinates are expectation values of physically interesting operators for the given quantum state. Our aim here is to build an adequate representation space for bipartite systems. We are guided by the relation of the standard Bloch sphere to a geometric object called the Hopf fibration of the $S^{3}$ hypersphere (identified to the spin $\frac{1}{2}$ Hilbert space). Since the two-qubits Hilbert space is the sevendimensional sphere $S^{7}$, which also allows for a Hopf fibration, it is tempting to mimic the Bloch sphere representation in this case. An a priori unexpected result is that the $S^{7}$ Hopf fibration is entanglement sensitive and therefore provides a kind of 'stratification' for the two-qubit states with respect to their entanglement content.

This paper is organized as follows. We first briefly recall known facts about the Bloch sphere representation and its relation to the $S^{3}$ Hopf fibration. We then proceed to the twoqubits states and recall what entanglement and Schmidt decomposition consist in. The $S^{7}$ Hopf fibration is then introduced and related to the two-qubits Hilbert space. Although several papers have appeared (especially in the recent period) which aimed at a geometrical analysis of qubits Hilbert space [2], we are not aware of an alternative use of the $S^{7}$ Hopf fibration in this context. As far as computation is concerned, going from the $S^{3}$ to the $S^{7}$ fibration merely amounts to replacing complex numbers by quaternions. This is why we give a brief introduction to quaternion numbers in the appendix. Note that using quaternions is not strictly necessary here, but they provide an elegant way to put the calculations into a compact form, and have (by nature) an easy geometrical interpretation.

\section{Single qubit, Bloch sphere and the $S^{3}$ Hopf fibration}

\subsection{The Bloch sphere representation}

A (single) qubit state reads

$$
|\Psi\rangle=\alpha|0\rangle+\beta|1\rangle \quad \alpha, \beta \in \mathbb{C} \quad|\alpha|^{2}+|\beta|^{2}=1 .
$$

In the spin $\frac{1}{2}$ context, the orthonormal basis $\{|0\rangle,|1\rangle\}$ is composed of two eigenvectors of the (say) $\sigma_{z}$ (Pauli spin) operator. A convenient way to represent $|\Psi\rangle$ (up to a global phase) is then provided by the Bloch sphere. The set of states exp $\mathrm{i} \varphi|\Psi\rangle(\varphi \in[0,2 \pi[)$ is mapped onto a point on $S^{2}$ (the usual sphere in $\mathrm{R}^{3}$ ) with coordinates $(X, Y, Z)$

$$
\begin{aligned}
& X=\left\langle\sigma_{x}\right\rangle_{\Psi}=2 \operatorname{Re}(\bar{\alpha} \beta) \\
& Y=\left\langle\sigma_{y}\right\rangle_{\Psi}=2 \operatorname{Im}(\bar{\alpha} \beta) \\
& Z=\left\langle\sigma_{z}\right\rangle_{\Psi}=|\alpha|^{2}-|\beta|^{2}
\end{aligned}
$$

where $\bar{\alpha}$ is the complex conjugate of $\alpha$. Recall also the relation between Bloch sphere coordinates and the pure state density matrix $\rho_{|\Psi\rangle}$ :

$$
\rho_{|\Psi\rangle}=\rho_{\exp i \varphi|\Psi\rangle}=|\Psi\rangle\langle\Psi|=\frac{1}{2}\left(\begin{array}{cc}
1+Z & X-\mathrm{i} Y \\
X+\mathrm{i} Y & 1-Z
\end{array}\right) .
$$

For mixed states, the density matrices are in one-to-one correspondence with points in the Bloch 'ball', the interior of the pure state Bloch sphere.

The single qubit Hilbert space is the unit sphere $S^{3}$ embedded in $R^{4}$. Indeed, writing $\alpha=x_{1}+\mathrm{i} x_{2}$ and $\beta=x_{3}+\mathrm{i} x_{4}$, the state normalization condition translates into $\sum x_{l}^{2}=1$ defining the $S^{3}$ sphere. In this space, the set of states exp i $\varphi|\Psi\rangle$ is a circle parametrized by $\varphi$. The projective Hilbert space is such that all states differing by a global phase are identified, 
and therefore corresponds to the above Bloch sphere. It can also be identified to the Hopf fibration basis as follows.

\subsection{The $S^{3}$ Hopf fibration}

In brief, a space is fibred if it has a subspace (the fibre) which can be shifted by a displacement, so that any point of the space belongs to one and only one fibre. For example, the Euclidean space $R^{3}$ can be seen not only as a fibre bundle of parallel straight lines, but also of parallel planes (the fibre need not be one dimensional). More precisely, a fibred space $E$ is defined by a (many-to-one) map from $E$ to the so-called 'base space' $B$, all points of a given fibre $F$ being mapped onto a single base point. In the preceding $R^{3}$ example, the base is either a plane cutting the whole set of parallel lines or, in the second case, a line cutting the set of parallel planes. Here, we are facing 'trivial fibrations', in the sense that the base $B$ is embedded in the fibred space $E$, the latter being faithfully described as the direct product of the base and the fibre: $R^{3}=R^{2} \times R$ or $R^{3}=R \times R^{2}$.

The simplest, and the most famous, example of a non-trivial fibration is the Hopf fibration of $S^{3}$ by great circles $S^{1}$ and base space $S^{2}$. For the qubit Hilbert space, the fibre represents the global phase degree of freedom, and the base $S^{2}$ is identified as the Bloch sphere. The standard notation for a fibred space is that of a map $E \stackrel{F}{\rightarrow} B$, which here reads $S^{3} \stackrel{S^{1}}{\rightarrow} S^{2}$. Its non-trivial character implies that $S^{3} \neq S^{2} \times S^{1}$. This non-trivial character translates into the known failure in ascribing consistently a definite phase to each representing point on the Bloch sphere.

To describe this fibration in an analytical form, we go back to the definition of $S^{3}$ as pairs of complex numbers $(\alpha, \beta)$ which satisfy $|\alpha|^{2}+|\beta|^{2}=1$. The Hopf map is defined as the composition of a map $h_{1}$ from $S^{3}$ to $R^{2}(+\infty)$, followed by an inverse stereographic map from $R^{2}$ to $S^{2}$ :

$$
\begin{aligned}
& \begin{array}{ccc}
h_{1}: & \longrightarrow & R^{2}+\{\infty\} \\
(\alpha, \beta) & \longrightarrow & C=\overline{\alpha \beta^{-1}}
\end{array} \quad \alpha, \beta \in \mathbb{C} \\
& \begin{array}{cccc}
h_{2}: & R^{2}+\{\infty\} & \longrightarrow & S^{2} \\
C & \longrightarrow & M(X, Y, Z) & X^{2}+Y^{2}+Z^{2}=1
\end{array}
\end{aligned}
$$

The first map $h_{1}$ clearly shows that the full $S^{3}$ great circle, parametrized by $(\alpha \operatorname{expi} \varphi, \beta \operatorname{expi} \varphi$ ) is mapped on the same single point with a complex coordinate $C$. Note that the complex conjugation, in the above definition for the Hopf map, $h_{1}$, is not necessary to represent a great circle fibration. It is used here on purpose to get an exact one-to-one relation with the above Bloch sphere coordinates and prepare the generalization to higher dimension discussed below. It is indeed a simple exercise to show that with $R^{2}$ cutting the unit radius $S^{2}$ along the equator and the north pole as the stereographic projection pole, the $S^{2}$ Hopf fibration base coordinates coincide with the above $S^{2}$ Bloch sphere coordinates.

Although there is nothing really new in this correspondence [3], it is probably poorly known in both communities (quantum optics and geometry). It is striking that the simplest non-trivial object of quantum physics, the two-level system, bears an intimate relation with the simplest non-trivial fibred space.

It is tempting to try to visualize the full $\left(S^{3}\right)$ Hilbert space with its fibre structure. This can be achieved by doing a (direct) stereographic map from $S^{3}$ to $R^{3}$ (nice pictures can be found in [3, 4]). Each $S^{3}$ circular fibre is mapped onto a circle in $R^{3}$, with an exceptional straight line, image of the unique $S^{3}$ great circle passing through the projection pole. The great circle arrangement is intricate, since each circular fibre threads all others in $S^{3}$. 


\section{Two qubits, entanglement and the $S^{7}$ Hopf fibration}

\subsection{The two-qubit Hilbert space}

We now proceed one step further, and investigate pure states for two qubits. The Hilbert space $\mathcal{E}$ for the compound system is the tensor product of the individual Hilbert spaces $\mathcal{E}_{1} \otimes \mathcal{E}_{2}$, with a direct product basis $\{|00\rangle,|01\rangle,|10\rangle,|11\rangle\}$. A two-qubit pure state reads

$$
\begin{aligned}
& |\Psi\rangle=\alpha|00\rangle+\beta|01\rangle+\gamma|10\rangle+\delta|11\rangle \\
& \text { with } \quad \alpha, \beta, \gamma, \delta \in \mathbb{C} \quad \text { and }|\alpha|^{2}+|\beta|^{2}+|\gamma|^{2}+|\delta|^{2}=1 .
\end{aligned}
$$

$|\Psi\rangle$ is said 'separable' if, at the price of possible basis changes in $\mathcal{E}_{1}$ and $\mathcal{E}_{2}$ separately, it can be written as a single product. As is well known, the separability condition reads $\alpha \delta=\beta \gamma$. A generic state is not separable, and is said to be 'entangled'. The Schmidt decomposition [5] implies that a suitable basis exists such that $|\Psi\rangle$ can be written as a sum of only two (biorthonormal) terms with real, positive coefficients. Note that the apparently obvious decomposition

$|\Psi\rangle=\cos \Omega\left(|0\rangle_{1} \otimes\left|u_{2}\right\rangle_{2}\right)+\sin \Omega\left(|1\rangle_{1} \otimes\left|v_{2}\right\rangle_{2}\right)$

with $\quad\left|u_{2}\right\rangle_{2}=\frac{1}{\cos \Omega}\left(\alpha|0\rangle_{2}+\beta|1\rangle_{2}\right) \quad\left|v_{2}\right\rangle_{2}=\frac{1}{\sin \Omega}\left(\gamma|0\rangle_{2}+\delta|1\rangle_{2}\right)$

$\cos \Omega=\left(|\alpha|^{2}+|\beta|^{2}\right)^{1 / 2} \quad$ and $\quad \sin \Omega=\left(|\gamma|^{2}+|\delta|^{2}\right)^{1 / 2} \quad \Omega \in[0, \pi / 2]$

is generically not a Schmidt decomposition since $\left\langle u_{2} \mid v_{2}\right\rangle$ need not vanish, a point to which we return below.

The $|\Psi\rangle$ normalization condition $|\alpha|^{2}+|\beta|^{2}+|\gamma|^{2}+|\delta|^{2}=1$ identifies $\mathcal{E}$ to the sevendimensional sphere $S^{7}$, embedded in $R^{8}$. It is therefore tempting to see whether or not the known $S^{7}$ Hopf fibration (with fibres $S^{3}$ and base $S^{4}$ ) can play any role in the Hilbert space description.

\subsection{The $S^{7}$ Hopf fibration}

The simplest way to introduce this fibration is to proceed along the same line as for the $S^{3}$ case, but using quaternions instead of complex numbers (see appendix). We write

$$
q_{1}=\alpha+\beta \mathbf{j} \quad q_{2}=\gamma+\delta \mathbf{j} \quad q_{1}, q_{2} \in \mathbb{Q}
$$

and a point (representing the state $|\Psi\rangle$ ) on the unit radius $S^{7}$ as a pair of quaternions $\left(q_{1}, q_{2}\right)$ satisfying $\left|q_{1}\right|^{2}+\left|q_{2}\right|^{2}=1$. The Hopf map from $S^{7}$ to the base $S^{4}$ is the composition of a map $h_{1}$ from $S^{7}$ to $R^{4}(+\infty)$, followed by an inverse stereographic map from $R^{4}$ to $S^{4}$ [4].

$$
\begin{aligned}
& \begin{array}{ccc}
h_{1}: & \longrightarrow & R^{4}+\{\infty\} \\
\left(q_{1}, q_{2}\right) & \longrightarrow & Q=\overline{q_{1} q_{2}^{-1}}
\end{array} \quad q_{1}, q_{2} \in \mathbb{Q} \\
& h_{2}: \begin{array}{ccc}
R^{4}+\{\infty\} & \longrightarrow & S^{4} \\
Q & \longrightarrow & M\left(x_{l}\right)
\end{array} \quad \sum_{l=0}^{l=4} x_{l}^{2}=1 .
\end{aligned}
$$

This Hopf fibration therefore reads $S^{7} \stackrel{S^{3}}{\rightarrow} S^{4}$. The base space $S^{4}$ is not embedded in $S^{7}$ : the fibration is again not trivial. The fibre is a unit $S^{3}$ sphere as can easily be seen by noting that the $S^{7}$ points $\left(q_{1}, q_{2}\right)$ and $\left(q_{1} q, q_{2} q\right)$, with $q$ a unit quaternion (geometrically a $S^{3}$ sphere), are mapped onto the same $Q$ value. A main difference with the previous $S^{3}$ Hopf fibration comes from the non-commutative nature of the quaternion product. The Hopf map $h_{1}^{\prime}$, defined by $Q=\overline{q_{2}^{-1} q_{1}}$ is distinct from $h_{1}$, while still corresponding to a fibration $S^{7} \stackrel{S^{3}}{\rightarrow} S^{4}$. 
One must stress that due to the tensor product nature of the two-qubit Hilbert space, the latter inherits a form of 'anisotropy' (in $S^{7}$ ) which translates into specific choices for the definition of the pair $\left(q_{1}, q_{2}\right)$, as discussed below.

Let us now look more closely at the base space $S^{4}$, by working out explicitly the Hopf map. The $h_{1}$ map leads to

$Q=\overline{q_{1} q_{2}^{-1}}=\frac{1}{\sin ^{2} \Omega}[\overline{(\alpha+\beta \mathbf{j})(\bar{\gamma}-\delta \mathbf{j})}]=\frac{1}{\sin ^{2} \Omega}\left(C_{1}+C_{2} \mathbf{j}\right)$

with $\quad C_{1}=(\bar{\alpha} \gamma+\bar{\beta} \delta) \quad C_{2}=(\alpha \delta-\beta \gamma) \quad$ and $\quad C_{1}, C_{2} \in \mathbb{C}$

Now comes the first striking result: the Hopf map is entanglement sensitive! Indeed, separable states satisfy $\alpha \delta=\beta \gamma$ and therefore map onto the subset of pure complex numbers in the quaternion field (both being completed by $\infty$ when $\sin \Omega=0$ ). Geometrically, this means that non-entangled states map from $S^{7}$ onto a two-dimensional planar subspace of the target space $R^{4}$. Note however that the latter property heavily depends on the chosen analytical form of the $h_{1}$ map (in other words, on the 'orientation' of the fibres with respect to $S^{7}$ ). A second interesting point concerns states whose Hopf map is confined in the two-dimensional subspace orthogonal to the previous one (such that $C_{1}=0$ ). This plane gathers the images of states with the above trivial Schmidt decomposition: indeed, $C_{1}=0$ implies $\left\langle u_{2} \mid v_{2}\right\rangle=0$.

The second map $h_{2}$ sends states onto points on $S^{4}$, with coordinates $x_{l}$, with $l$ running from 0 to 4 . With the inverse stereographic pole located on the $S^{4}$ 'north' pole $\left(x_{0}=+1\right)$, and the target space $R^{4}$ cutting $S^{4}$ along the equator, we get the following coordinate expressions

$$
\begin{aligned}
& x_{0}=\cos 2 \Omega=\left|q_{1}\right|^{2}-\left|q_{2}\right|^{2} \\
& x_{1}=\sin 2 \Omega S\left(Q^{\prime}\right)=2 \operatorname{Re}(\bar{\alpha} \gamma+\bar{\beta} \delta) \\
& x_{2}=\sin 2 \Omega V_{\mathbf{i}}\left(Q^{\prime}\right)=2 \operatorname{Im}(\bar{\alpha} \gamma+\bar{\beta} \delta) \\
& x_{3}=\sin 2 \Omega V_{\mathbf{j}}\left(Q^{\prime}\right)=2 \operatorname{Re}(\alpha \delta-\beta \gamma) \\
& x_{4}=\sin 2 \Omega V_{\mathbf{k}}\left(Q^{\prime}\right)=2 \operatorname{Im}(\alpha \delta-\beta \gamma)
\end{aligned}
$$

with $\sin 2 \Omega=2\left|q_{1} \| q_{2}\right| . Q^{\prime}$ is the normalized image of the $h_{1}$ map $\left(Q^{\prime}=Q /|Q|\right), S\left(Q^{\prime}\right)$ and $V_{\mathbf{i}, \mathbf{j}, \mathbf{k}}\left(Q^{\prime}\right)$ are, respectively, the scalar and vectorial parts of $Q^{\prime}$ (see appendix). We recalled above that the $X, Y$ and $Z$ Bloch sphere coordinates are expectation values of the spin operator $\sigma$ in the one-qubit state $|\Psi\rangle$ (equation (1)). It happens that the $x_{l}$ coordinates are also expectation values of simple operators in the two-qubit state (equation (5)).

An obvious one is $x_{0}$ which corresponds to $\left\langle\sigma_{z} \otimes I d\right\rangle_{\Psi}$. Indeed,

$\left\langle\sigma_{z} \otimes I d\right\rangle_{\Psi}=(\bar{\alpha}, \bar{\beta}, \bar{\gamma}, \bar{\delta})\left(\begin{array}{cccc}1 & 0 & 0 & 0 \\ 0 & 1 & 0 & 0 \\ 0 & 0 & -1 & 0 \\ 0 & 0 & 0 & -1\end{array}\right)\left(\begin{array}{l}\alpha \\ \beta \\ \gamma \\ \delta\end{array}\right)=\left|q_{1}\right|^{2}-\left|q_{2}\right|^{2}=\cos 2 \Omega=x_{0}$.

The next two coordinates are also easily recovered as

$$
\begin{aligned}
& x_{1}=\left\langle\sigma_{x} \otimes I d\right\rangle_{\Psi}=(\bar{\alpha}, \bar{\beta}, \bar{\gamma}, \bar{\delta})\left(\begin{array}{cccc}
0 & 0 & 1 & 0 \\
0 & 0 & 0 & 1 \\
1 & 0 & 0 & 0 \\
0 & 1 & 0 & 0
\end{array}\right)\left(\begin{array}{l}
\alpha \\
\beta \\
\gamma \\
\delta
\end{array}\right)=2 \operatorname{Re}(\bar{\alpha} \gamma+\bar{\beta} \delta) \\
& x_{2}=\left\langle\sigma_{y} \otimes I d\right\rangle_{\Psi}=(\bar{\alpha}, \bar{\beta}, \bar{\gamma}, \bar{\delta})\left(\begin{array}{cccc}
0 & 0 & -\mathrm{i} & 0 \\
0 & 0 & 0 & -\mathrm{i} \\
\mathrm{i} & 0 & 0 & 0 \\
0 & \mathrm{i} & 0 & 0
\end{array}\right)\left(\begin{array}{l}
\alpha \\
\beta \\
\gamma \\
\delta
\end{array}\right)=2 \operatorname{Im}(\bar{\alpha} \gamma+\bar{\beta} \delta) .
\end{aligned}
$$


The remaining two coordinates, $x_{3}$ and $x_{4}$, are also recovered as expectation values of an operator acting on $\mathcal{E}$, but in a more subtle way. Let us define $\mathbf{J}$ as the (antilinear) 'conjugator', an operator which takes the complex conjugate of all complex numbers involved in an expression (here acting on the left-hand side in the scalar product below) and then form the antilinear operator $\mathbf{E}$ (for 'entanglor'): $\mathbf{E}=-\mathbf{J}\left(\sigma_{y} \otimes \sigma_{y}\right)$. One finds

$$
\begin{aligned}
& x_{3}=\operatorname{Re}\langle\mathbf{E}\rangle_{\Psi} \\
& x_{4}=\operatorname{Im}\langle\mathbf{E}\rangle_{\Psi} .
\end{aligned}
$$

Note that $\langle\mathbf{E}\rangle_{\Psi}$ vanishes for non-entangled states, and takes its maximal norm (equals to 1) for maximally entangled states, hence the name entanglor. Such an operator, which is nothing but the time reversal operator for two spins $\frac{1}{2}$, has already been largely used in quantifying entanglement [6], with a quantity $c$, called the 'concurrence', which here is defined as $c=2\left|C_{2}\right|$.

\subsection{Discussion}

Let us now see what has been gained in fibrating the $\mathcal{E}$ two-qubit Hilbert space. It is clear that at some point, we will need to focus on the 'projective' Hilbert space, taking into account the global phase freedom. It is nevertheless interesting to first stay at the Hilbert space level, and foliate the base space along constant values of simple operators expectation values. The simplest thing to do is to fix $\left\langle\sigma_{z} \otimes I d\right\rangle_{\Psi}$, e.g. $x_{0}$. Let us start from the $S^{4}$ base north pole, $x_{0}=1$, which is the image of the states

$$
\Psi_{Q=\infty}=|0\rangle_{1} \otimes\left(\alpha|0\rangle_{2}+\beta|0\rangle_{2}\right) .
$$

Decreasing the $x_{0}$ values, we get a sequence of 'horizontal' $S^{3}$ spheres of radius $\sin 2 \Omega$, denoted by $S_{\Omega}^{3}$. This is nothing but the generalization in higher dimension of the standard horizontal 'parallel' circles which foliates $S^{2}$. As is well known, $S^{3}$ can also be foliated by nested tori, with two opposite nested great circles sitting on the orthogonal two planes $\left(x_{1}, x_{2}\right)$ and $\left(x_{3}, x_{4}\right)$. As already noted, states where $x_{3}=x_{4}=0$ are non-entangled states. Maximally entangled states (MES) correspond to $x_{0}=x_{1}=x_{2}=0$. As far as the geometric description is concerned, it is also useful to define $\Omega$ MES, which are states with fixed values of $\left\langle\sigma_{z} \otimes I d\right\rangle_{\Psi}$ (e.g. constant $\left.x_{0}\right)$ and, with this constraint, maximal entanglement (e.g. maximal concurrence). Usual MES are nothing but $\frac{\pi}{4} \mathrm{MES}$, and we show below that all $\Omega$ MES manifolds share the same topology, except for the extremal values $\Omega=0$ and $\Omega=\pi / 2$, which are separable states.

Let us now inverse the Hopf map and get the general expression for a state (a $S^{7}$ point) which is sent to $Q$ by the $h_{1}$ map. Such a state, denoted by $\Psi_{Q}$ reads (given as a pair of quaternions)

$$
\Psi_{Q}=(\cos \Omega \exp (-\theta \mathbf{t} / 2) q, \sin \Omega \exp (\theta \mathbf{t} / 2) q)
$$

where $\cos \theta=x_{1} / \sin 2 \Omega=S\left(Q^{\prime}\right), q$ is a unit quaternion which spans the $S^{3}$ fibre and $\mathbf{t}$ is the following unit purely imaginary quaternion:

$$
\mathbf{t}=\left(\mathbf{V}_{\mathbf{i}}\left(Q^{\prime}\right) \mathbf{i}+\mathbf{V}_{\mathbf{j}}\left(Q^{\prime}\right) \mathbf{j}+\mathbf{V}_{\mathbf{k}}\left(Q^{\prime}\right) \mathbf{k}\right) / \sin \theta
$$

3.3.1. Separable states. In the non-entangled case, $Q$ is now a complex number and the above expression simplifies to

$$
\Psi_{Q}=(\cos \Omega \exp (-\theta \mathbf{i} / 2) q, \sin \Omega \exp (\theta \mathbf{i} / 2) q) .
$$


The projective Hilbert space for two non-entangled qubits $A$ and $B$ is expected to be the product of two two-dimensional spheres $S_{A}^{2} \times S_{B}^{2}$, each sphere being the Bloch sphere associated with the given qubit. This property is clearly displayed here. The unit $S^{4}$ base space reduces in a unit $S^{2}$ sphere (since $x_{3}=x_{4}=0$ ), which is nothing but the Bloch sphere for the first qubit, as seen in the coordinate expressions, $\left\langle\sigma_{z, x, y} \otimes I d\right\rangle_{\Psi}$, for $x_{0}, x_{1}$ and $x_{2}$ respectively. This $S^{2}$ sphere is the aggregate of parallel circles, of radii $\sin 2 \Omega$ in the plane $\left(x_{1}, x_{2}\right)$, each one on a parallel $S_{\Omega}^{3}$ sphere, and with the extremal poles corresponding to $\Omega=0$ and $\Omega=\pi / 2$. For the second qubit Bloch sphere, one needs to define a coordinate system on the fibres. We choose the two orthogonal states $|0\rangle_{Q}$ and $|1\rangle_{Q}$, respectively, corresponding to $q=1$ and $q=j$ in the above expression for $\Psi_{Q}$. They read, in the initial $\mathcal{E}$ product state basis

$$
\begin{aligned}
|0\rangle_{Q} & =\left(\cos \Omega \exp (-\mathrm{i} \theta / 2)|0\rangle_{1}+\sin \Omega \exp (\mathrm{i} \theta / 2)|1\rangle_{1}\right) \otimes|0\rangle_{2} \\
|1\rangle_{Q} & =\left(\cos \Omega \exp (-\mathrm{i} \theta / 2)|0\rangle_{1}+\sin \Omega \exp (\mathrm{i} \theta / 2)|1\rangle_{1}\right) \otimes|1\rangle_{2} .
\end{aligned}
$$

A generic state $\Psi_{Q}$ in the $S^{3}$ fibre reads $\left|\Psi_{Q}\right\rangle=a|0\rangle_{Q}+b|1\rangle_{Q}$, with $a, b \in \mathbb{C}$, $|a|^{2}+|b|^{2}=1$. We can now iterate the fibration process on the fibre itself and get the (Hopf fibration base)-(Bloch sphere) coordinates for this two-level system. It is now a simple exercise to verify that the three real coordinates obtained are precisely $\left\langle I d \otimes \sigma_{x, y, z}\right\rangle_{\Psi}$, that is, the second qubit Bloch sphere.

In summary, for non-entangled qubits, the $S^{7}$ Hopf fibration with base $S^{4}$ and fibre $S^{3}$ simplifies to the simple product of an $S^{2}$ subsphere of the base (the first qubit Bloch sphere) and a second $S^{2}$ (the second qubit Bloch sphere) obtained as the base of an $S^{3}$ Hopf fibration applied to the fibre itself. Let us stress that this last iterated fibration is necessary to take into account the global phase of the two-qubit system.

The fact that these two $S^{2}$ spheres play a symmetrical role (although one is related to the base and the other to the fibre) can be understood in the following way. We grouped together $\alpha$ and $\beta$ on one hand, and $\gamma$ and $\delta$ on the other hand, to form the quaternions $q_{1}$ and $q_{2}$, and then defined the Hopf map $h_{1}$ as the ratio of these two quaternions (plus a complex conjugation). Had we grouped $\alpha$ and $\gamma$, and $\beta$ and $\delta$, to form two new quaternions, and used the same definition for the Hopf map, we would also get an $S^{7}$ Hopf fibration, but differently oriented. We leave it as an exercise for the reader to compute the base and fibre coordinates in this case. The net effect is to interchange the role of the two qubits: the second qubit Bloch sphere is now part of the $S^{4}$ base, while the first qubit Bloch sphere is obtained from the $S^{3}$ fibre.

3.3.2. Entangled states. As expected, the entangled case is more complicated. From an analytical point of view, this is related to the fact that the pure imaginary quaternion $\mathbf{t}$ does not reduce to the standard $\mathbf{i}$ imaginary unit. Let us focus first on the MES. They correspond to the complex number $C_{2}$ having a maximal norm $\frac{1}{2}$. This in turn implies that the Hopf map base reduces to a unit circle in the plane $\left(x_{3}, x_{4}\right)$, parametrized by the unit complex number $2 C_{2}$. The projective Hilbert space for these MES is known to be $S^{3} / Z_{2}$, an $S^{3}$ sphere with opposite points identified [2] (this is linked to the fact that all MES can be related by a local operation on one subsystem, since $S^{3} / Z_{2}=S O(3)$ ). In order to recover this result in the present framework, one can follow the trajectory of a representative point on the base and the fibre while the state is multiplied by an overall phase $\exp (\mathrm{i} \varphi)$. The expression for $C_{2}(=\alpha \delta-\beta \gamma)$ shows that the point on the base turns by twice the angle $\varphi$. Only when $\varphi=\pi$ does the corresponding state belong to the same fibre (e.g. maps onto the same value on the base). The fact that the fibre is an $S^{3}$ sphere and this two-to-one correspondence between the fibre and the base under a global phase change, explains the $S^{3} / Z_{2}$ topology for the MES projective Hilbert space. 
In this case, $\Psi_{Q}$ reads

$$
\frac{1}{\sqrt{2}}\left(\exp \left(-\pi C_{2} \mathbf{j} / 2\right) q, \exp \left(\pi C_{2} \mathbf{j} / 2\right) q\right) \text {. }
$$

The standard four Bell states are recovered with $q=(1 \pm \mathbf{j}) / \sqrt{2}$ and $C_{2}= \pm \frac{1}{2}$.

Now, it is clear that the previous discussion can be repeated for any $\Omega$ MES, which have therefore the same $S^{3} / Z_{2}$ topology, and reads

$$
\left(\cos \Omega \exp \left(-\frac{\pi}{4} \frac{C_{2}}{\left|C_{2}\right|} \mathbf{j}\right) q, \sin \Omega \exp \left(\frac{\pi}{4} \frac{C_{2}}{\left|C_{2}\right|} \mathbf{j}\right) q\right) .
$$

Finally, we consider generic two-qubit states, which are neither separable nor maximally entangled. For each value of $\Omega$ (e.g. of $\left\langle\sigma_{z} \otimes I d\right\rangle_{\Psi}$ ), these states are mapped (through the $h_{1}$ map) onto a torus of the $S_{\Omega}^{3}$ torus foliation. Such a torus reduces to a circle of radius $2\left|C_{1}\right|$ for the projective version. To each point of these circles is again associated an $S^{3} / Z_{2}$ manifold in the fibre part.

3.3.3. A tentative generalization of the Bloch sphere representation. We are now led to consider a tentative (and partly well known, see below) generalization of the Bloch sphere for the two-qubit projective Hilbert space. Clearly, the present Hopf fibration description suggests a splitting of the representation space in the product of base and fibres subspaces. Of the base space $S^{4}$, we propose to only keep the first three coordinates,

$$
\left(x_{0}, x_{1}, x_{2}\right)=\left(\left\langle\sigma_{z} \otimes I d\right\rangle_{\Psi},\left\langle\sigma_{x} \otimes I d\right\rangle_{\Psi},\left\langle\sigma_{y} \otimes I d\right\rangle_{\Psi}\right) .
$$

All states map into a standard ball $B^{3}$ of radius 1 , where the set of separable states forms the $S^{2}$ boundary (the usual first qubit Bloch sphere), and the centre corresponds to maximally extended states. Concentric spherical shells around the centre correspond to states of equal concurrence $c$ (maximal at the centre, zero on the surface), the radius of the spherical shell being equal to $\sqrt{1-c^{2}}$. The idea of slicing the two-qubit Hilbert space into manifolds of equal concurrence is not new [2]. What is interesting here is that under the Hopf map (and a projection onto the 3D subspace of the base spanned by the first three coordinates), these manifolds transform into concentric $S^{2}$ shells which fill the unit ball. We must now describe which subset of the $S^{3}$ fibres has to be attached to each point in this base-related unit ball. Looking into the previous discussions, it is clear that it is an $S^{3} / Z_{2}$ space for each interior point, and an $S^{2}$ space (the second qubit Bloch sphere) for the boundary of the unit ball.

The Bloch ball single qubit mixed state representation was recalled above. The centre of the Bloch ball corresponds to maximally mixed states. The reader might be surprised to find here (in the two-qubit case) a second unit-radius ball, with MES at the centre. There is no mystery here, and it corresponds to a known relation between partially traced two-qubit pure states and a one-qubit mixed state. The partially traced density matrix $\rho_{1}$ is in fact deeply related to the $S^{7}$ Hopf map:

$$
\rho_{1}=\left(\begin{array}{cc}
\left|q_{1}\right|^{2} & \overline{C_{1}} \\
C_{1} & \left|q_{2}\right|^{2}
\end{array}\right)=\frac{1}{2}\left(\begin{array}{cc}
1+x_{0} & x_{1}-\mathbf{i} x_{2} \\
x_{1}+\mathbf{i} x_{2} & 1-x_{0}
\end{array}\right)
$$

with unit trace and $\operatorname{det} \rho_{1}=\left|C_{2}\right|^{2}$. $\rho_{1}$ represents a pure state density matrix whenever $C_{2}$ vanishes (the separable case), and allows for a unit Bloch sphere (that associated to the first qubit). It is assimilated to a mixed state density matrix as soon as $\left|C_{2}\right|>0$ (and an entangled state for the two-qubit state). The other partially traced density matrix $\rho_{2}$ is related to the other $S^{7}$ Hopf fibration which was discussed above. With all these in mind, one also expects 
a simple generic relation between the Hopf map and the Schmidt decomposition. Indeed the two common eigenvalues of $\rho_{1}$ and $\rho_{2}$ read

$$
\lambda_{ \pm}=\frac{1+\sqrt{1-4\left|C_{2}\right|^{2}}}{2}=\frac{1+\sqrt{1-c^{2}}}{2}
$$

from which we get the Schmidt weights $\cos \frac{\varepsilon}{2}=\sqrt{\lambda_{+}}$and $\sin \frac{\varepsilon}{2}=\sqrt{\lambda_{-}}$. The Schmidt states are easily obtained from the diagonalization of $\rho_{1}$ and $\rho_{2}$. Their decomposition in the original product state basis can therefore also be given in term of the Hopf map parameters. As an example, the two (un-normalized) states for the first qubit simply read

$$
\left|\varphi_{ \pm}\right\rangle=\overline{C_{1}}|0\rangle_{1}+\left(\lambda_{ \pm}-\left|q_{1}\right|^{2}\right)|1\rangle_{1}
$$

\section{Conclusion}

Our main goal in this paper was to provide a geometrical representation of the two-qubit Hilbert space pure states. We hope to have convinced the reader that the $S^{7}$ Hopf fibration plays a natural role in this case: it provides an adapted 'skeleton' for preparing a meaningfull slicing of the full Hilbert space. It is rather fascinating that the two main Hopf fibrations (of $S^{3}$ and $S^{7}$ ) are so well related to the two important ingredients of the quantum objects, which are the global phase freedom and entanglement.

Going further, one may ask two natural questions. The first concerns the geometry of two-qubit mixed states, and the second concerns the generalization to more than two qubits. Note that these two questions are not necessarily completely independent, as we just saw in the preceding paragraph. Up to now, we have mainly focused on the second question, and addressed the three-qubit case [7]. Indeed, the Hilbert space is now $S^{15}$, which also allows for a Hopf fibration, with an $S^{8}$ base and $S^{7}$ fibres. From an analytical point of view, the Hopf map takes the same form, but quaternions need to be replaced by 'octonions'. This is not a harmless change, since octonion algebra is not only non-commutative but also non-associative (for instance, forbidding a matrix representation). But this analysis can be done, and provides interesting insights into the Hilbert space geometry. Note that in the three Hopf fibrations sequence $\left(S^{15}, S^{7}, S^{3}\right)$, the fibre in the larger dimensional space is the full space in next case. This offers the possibility of further nesting the fibrations, a possibility that we already used in the present analysis of a two-qubit separable case.

\section{Appendix. Quaternions}

Quaternions are usually presented with the imaginary units $\mathbf{i}, \mathbf{j}$ and $\mathbf{k}$ in the form $q=x_{0}+x_{1} \mathbf{i}+x_{2} \mathbf{j}+x_{3} \mathbf{k} \quad x_{0}, x_{1}, x_{2}, x_{3} \in \mathbb{R} \quad$ with $\quad \mathbf{i}^{2}=\mathbf{j}^{2}=\mathbf{k}^{2}=\mathbf{i j k}=-1$

with the latter 'Hamilton' relations defining the quaternion multiplication rules which are noncommutative. They can also be defined equivalently, using the complex numbers $c_{1}=x_{0}+x_{1} \mathbf{i}$ and $c_{2}=x_{2}+x_{3} \mathbf{i}$, in the form $q=c_{1}+c_{2} \mathbf{j}$.

The conjugate of a quaternion $q$ is $\bar{q}=x_{0}-x_{1} \mathbf{i}-x_{2} \mathbf{j}-x_{3} \mathbf{k}=\overline{c_{1}}-c_{2} \mathbf{j}$ and its squared norm reads $N_{q}^{2}=q \bar{q}$.

Another way in which $q$ can be written is as the sum of a scalar part $S(q)$ and a vectorial part $\mathbf{V}(q)$ :

$$
q=S(q)+\mathbf{V}(q) \quad S(q)=x_{0} \quad \mathbf{V}(q)=x_{1} \mathbf{i}+x_{2} \mathbf{j}+x_{3} \mathbf{k}
$$

with the relations

$$
S(q)=\frac{1}{2}(q+\bar{q}) \quad \mathbf{V}(q)=\frac{1}{2}(q-\bar{q}) .
$$


A quaternion is said to be real if $\mathbf{V}(q)=0$, and purely imaginary if $S(q)=0$. We shall also write $\mathbf{V}_{\mathbf{i}, \mathbf{j}, \mathbf{k}}(q)$ for the component of $\mathbf{V}(q)$ along $\mathbf{i}, \mathbf{j}, \mathbf{k}$. Finally, for complex numbers, a quaternion can be noted in an exponential form as

$$
q=|q| \exp \varphi \mathbf{t}=|q|(\cos \varphi+\sin \varphi \mathbf{t})
$$

where $\mathbf{t}$ is a unit purely imaginary quaternion. When $\mathbf{t}=\mathbf{i}$, usual complex numbers are recovered. Note that quaternion multiplication is non-commutative so that

$$
\exp \varphi \mathbf{t} \exp \lambda \mathbf{u}=\exp (\varphi \mathbf{t}+\lambda \mathbf{u})
$$

only if $\mathbf{t}=\mathbf{u}$.

\section{Acknowledgment}

It is a pleasure for one of the authors (RM) to acknowledge enlightening discussions about quantum information with $\mathrm{O}$ Cohen and B Griffiths.

The content of this paper was presented at the National Congress of the French Physical Society, Strasbourg, 9-13 July 2001 (unpublished).

While completing the present written version, we came across a very interesting preprint by I Bengtsson, J Brännlund and K Zyczkowski (quant-phys/0108064) on the geometry of qubits projective Hilbert space, with $S^{3}$ Hopf fibrations occasionally used to describe submanifolds. Besides a different perspective in slicing the Hilbert space, a main difference with our work is the present use of the $S^{7}$ Hopf fibration applied to the whole space.

\section{References}

[1] Bouwmeester D, Eckert A and Zeilinger A 2000 The Physics of Quantum Information (Berlin: Springer)

[2] Kus M and Zyczkowski K 2001 Phys. Rev. A 63 032307, and references therein

[3] Urbanke H 1991 Am. J. Phys. 5953

[4] Sadoc J F and Mosseri R 1999 Geometric Frustration (Cambridge: Cambridge University Press)

[5] Peres A 1993 Quantum Theory: Concepts and Methods (Dordrecht: Kluwer)

[6] Wootters W K 1998 Phys. Rev. Lett. 802245

[7] Mosseri R 2001 in preparation 